(S. 115) Heißt das für hiesige Intellektuelle eine Industrialisierung zu unterstützen, die nicht auch bürgerliche Freiheitsrechte (und wenn es auch "nur" solche sind) mit sich bringt? Hat man dann als Intellektueller nicht Verrat begangen, zwar nicht an seiner Klasse (mit allen Privilegien), aber doch an seinen emanzipatorischen Zielen? Die Frage der Ethik läßt sich nun mal nicht ausklammern.

$\mathrm{Da}$ eine andere Form menschlicher Arbeit als jene der "kapitalistischen Produktivkraftstruktur" in unserer Gesellschaft möglich werden könne, erwähnt Kößler am Ende des Artikels: "In immer wieder verdrehter Form" wird diese Frage von der "Energiedebatte" und der "Wachstumsdebatte" (S. 116) aufgeworfen.

An diesem Punkt wird deutlich, daß ohne ein gewisses Maß an Praxis die Theoriebildung haltlos in der Luft hängt. So ist auch das vorliegende Buch nicht der Anfang der neuen Sozialismusdebatte; neue, sozialistische Vorstellungen werden sich nur im Zusammenhang mit einer neuen gesellschaftlichen Praxis entwickeln. Doch dieses Buch trägt dazu bei, alte linke Denkschablonen zu Grabe zu tragen, die den Blick auf eine solche Praxis verstellen können.

Walter Aschmoneit

\title{
Joseph Tharamangalam
}

Agrarian class conflict. The political mobilization of agricultural labourers in Kuttanad, South India.

Vancouver, London: University of British Columbia Press 1981, 123 S., $\$ 19.00$

Bei der Studie von J. Tharamangalam handelt es sich um die leicht überarbeitete Fassung einer in der York University eingereichten Dissertation. Die Feldforschung wurde 1974/5 durchgeführt. Kuttanad (in Kerala) gehört mit zu den "unruhigsten“ ländlichen Reis-produzierenden Distrikten in Indien, mit einer hohen Bevölkerungsdichte und mit einem überproportionalen Anteil sowohl an Landlosen als auch an Alphabeten und inzwischen schließlich mit gut organisierten Landarbeitergewerkschaften. Die Untersuchung stellt sich die Aufgabe, "die Mobilisierung und den organisierten Kampf der Landarbeiter in Kuttanad für höhere Löhne, bessere Arbeitsbedingungen und, vor allem Menschenwürde" zu studieren (S. 4). Allerdings liegt der Schwerpunkt der Darstellung auf der Agrar- und Wirtschaftssituation, den Arbeitsbeziehungen, der Genese und Struktur der Landarbeiterschaft im Distrikt. Dabei wird interessantes Material zusammengestellt und analysiert, obwohl der Autor in seinen Ergebnissen nicht unbedingt über bekannte oder ohnehin plausible Erklärungsmuster hinausgelangt. Er führt so die hohe Mobilisierungsbereitschaft auf die hohe Bevölkerungs- und Kommunikationsdichte, die Nähe zu einer großen Stadt (Alleppey), schließlich auf den schnellen sozialen Wandel (hin zur kapitalistischen Landwirtschaft) und den dabei auftretenden Frustrationen und auf "das Vorhandensein und der Verfügbarkeit einer Partei mit einem angemessenen 
Ideologie und Strategie, die unzufriedenen Arbeiter zu organisieren" (gemeint ist die CPI (ML), S. 92 zurück. Letztere habe den Landarbeitern Richtung und Organisation sowie rechtlichen, finanziellen und physischen Schutz gegeben und unter ihrem Dach Allianzen mit anderen Klassen und Gruppen ermöglicht und unterhalten. Obwohl auch andere Parteien sich "ihre" Gewerkschaften zu gründen verstanden, was zu einer (wenn auch ungleichen) Spaltung der Landarbeiterbewegung geführt hat, scheint diese im Ganzen doch recht erfolgreich gewesen zu sein: Die relativ hohen Löhne (verglichen mit dem Landesdurchschnitt), die Durchsetzung geregelter Arbeitsbedingungen und der erfolgreiche Widerstand gegen eine drohende Mechanisierungswelle sind jedenfalls durchaus Indikatoren in diese Richtung. Dem steht allerdings auch hier gegenüber, daß - wie im ganzen übrigen Land - auch in Kuttanad sich die allgemeinen sozialen und ökonomischen Bedingungen für die Armutsgruppen noch weiter verschlechtert haben.

Obwohl der Autor der Landarbeiterschaft attestiert, (inzwischen) ein Klassenbewußtsein entwickelt zu haben, "sich in eine organisierte, militante und kämpferische Klasse 'für sich' entwickelt zu haben", sind nach ihm in den siebziger Jahren dennoch Desintegrationstendenzen in der (kommunistischen) Landarbeitergewerkschaft zu beobachten. Diese organisiere nur noch wenige praktische Programme, an denen die Arbeiter partizipieren würden. Sie würde daher unter diesen Umständen allmählich in eine sich selbstbedienende Bürokratie degenerieren.

Als Ursache dafür diagnostiziert der Autor, daß auch die CPI (ML) und ihre Gewerkschaft sich in das System integriert hätten und dort mit ihren gewerkschaftlichen Zielen ("höhere Löhne") an ihre Grenzen gestoßen seien. Er stellt abschließend fest: "They have little to offer the workers, however, except their rhetoric and a vague promise that if and when they are elected to power they will industrialize Kuttanad and create more jobs ... Their vision, at least for the foreseeable future, goes only as far as an ideal farmerlabour relationship; it does not extend to the abolition of that relationship and to the restructuring of society" (S. 97/98). Trotz dieser Schlußfolgerungen ist die Darstellung über die Landarbeitergewerkschaften eher spärlich in ihren Aussagen über organisationssoziologische Fragen und mehr historisch und ereignisgeschichtlich geschrieben. Die Studie dürfte dennoch für Regionalspezialisten von Interesse sein.

Rolf Hanisch

Giselher Foeth/Gottfried Zieger

Investitionen in Lateinamerika. Internationale Verträge und nationale Bestimmungen Dokumente, Band 45, Alfred Metzner Verlag Frankfurt a. M. 1979, 609 S., DM 95,-

Es handelt sich um eine sehr informative, sorgfältig recherchierte und plausibel kommentierte Arbeit eines ehemaligen wissenschaftlichen Referenten am Göttinger Institut für Völkerrecht. Dr. iur. Giselher Foeth - "seit 1976 im internationalen Finanzierungs- 